\title{
Le covoiturage domicile-travail : De l'approche technico-économique classique à une approche comportementale
}

\section{Commuters ridesharing: contribution of a behavioral economic approach}

\author{
Jean-Marc Josset \\ (C) IFSTTAR et Éditions NecPlus 2015
}

\begin{abstract}
Résumé Le covoiturage est souvent cité comme solution à la saturation des transports urbains mais reste une pratique marginale dans la population. Nous montrons dans ce papier qu'une approche comportementale permet la prise en compte de facteurs sociologiques et psychologiques qui constituent des freins majeurs à l'adoption de cette pratique. Nous en déduisons plusieurs hypothèses sur le passage à l'échelle, les incitations et la confiance que nous proposons d'explorer par des expérimentations de terrains.
\end{abstract}

Mots clés covoiturage · économie comportementale . expérimentation

\begin{abstract}
Ridesharing is often viewed as a way to solve urban transport crisis but fails to become an effective widespread practice in the population. We show in this paper that a behavioral approach allows the consideration of sociological and psychological factors which constitute major challenges to the adoption of this practice. We deduct several hypotheses for promoting massification, finding incentives and enabling trust that we propose to explore with field experiments.
\end{abstract}

Keywords Rideshharing - Behavioral Economics ·

Experiment

\section{Introduction}

D'après un rapport de l'Académie des sciences américaines [1] l'espace urbain mondial va tripler entre 2000 et 2030. En effet, ces décennies sont celles de l'explosion démographique : 8,5 milliards de terriens en 2030 dont 5 milliards de citadins. L'urbanisme métropolitain s'impose comme unique modèle de développement. Chaque jour on construit l'équivalent de la ville de Paris, et ses conséquences en

Jean-Marc Josset $(\bowtie)$

Doctorant en économie

Université Paris-Sud, RITM (Réseaux, Innovation, Territoires et

Mondialisation)

e-mail : jean-marc.josset@u-psud.fr externalités négatives : pollution, encombrements, sécurité. La société Inrix prévoit que le coût des encombrements pour la France devrait s'élever à 22 milliards d'euros en $2030^{1}$.

Les infrastructures nécessaires pour accompagner les besoins de déplacement en zones urbaines, à commencer par les transports en commun, nécessitent des investissements massifs et des délais de réalisations inadaptés à l'urgence de la situation. La question de leur financement est d'ailleurs posée : le World Economic Forum note dans son rapport 2012-2014 un déficit d'un trillion de dollars par an pour le développement des infrastructures nécessaires ${ }^{2}$.

Le comportement des usagers est donc devenu un sujet d'intérêt majeur, avec en première ligne le covoiturage, qui présente l'intérêt d'allier la flexibilité de la voiture avec le potentiel offert par les places-passager, généralement inoccupées.

Mais malgré l'engouement sans cesse renouvelé par les apports des nouvelles technologies comme le GPS, et quelques cas d'école (les HOV lanes $^{3}$, Blablacar ${ }^{4}$, Uber $^{5}$ ), le covoiturage reste une pratique marginale, et la multiplication des initiatives des collectivités locales et des entreprises dans le cadre des Plan de déplacement entreprise $(\mathrm{PDE})^{6}$ ne débouchent que sur des résultats décevants.

\footnotetext{
${ }^{1}$ Voir communiqué de presse sur le site : http://www.inrix.com/press/embouteillages-une-facture-cumulee-deplus-de-350-milliards-deuros-pour-la-france-sur-les-16-prochainesannees/ consulté le 10 février 2015.

${ }^{2}$ WEF Global agenda Concil on infrastructure 2012-2014 : http://www3.weforum.org/docs/GAC/2013/Connect/WEF_GAC_ Infrastructure_2012-2014_Connect.pdf

${ }^{3}$ High Occupancy Vehicule : voies de circulations réservées, selon le cas aux voitures avec un, deux ou trois passagers.

${ }^{4}$ Sur le marché du covoiturage, le succès exceptionnel de la société Blablacar est sans doute dû à son positionnement sur les trajets longue distance et sa proposition de valeur essentiellement financière. Voir typologie dans la suite de l'article.

${ }^{5}$ Les services de taxi accessibles par internet mobile comme Uber ou Lift aux États-Unis se positionnent sur le « covoiturage payant » ouvert à des non professionnels.

${ }^{6}$ Dans le cadre d'un covoiturage organisé par les entreprises : $0,8 \%$ des employés inscrits en moyenne dont $10 \%$ seulement «passent à l'acte » Ademe [2].
} 
Quels sont les freins qui empêchent cette pratique de se développer et comment les lever ? Nous constatons la difficulté à prendre en compte les aspects sociaux et psychologiques des pratiques de covoiturage dans les modèles d'études. Faut-il pour autant abandonner toute prétention à une approche économique du sujet comme semblent le suggérer Schuldiner et Schuldiner [3] ?

Les propositions de la littérature se concentrent généralement sur une optimisation de la problématique de coordination conducteur/passager [4], [5]. Cette approche repose implicitement sur un présupposé d'agents rationnels (homo oconomicus) qui ne permet pas de prendre en compte les freins sociologiques et psychologiques, pourtant relevés par de nombreuses enquêtes, principalement la motivation et la confiance des usagers [6], [7], [8].

Pour répondre à ces points, nous proposons de définir un cadre expérimental qui permette d'explorer les conditions du développement du covoiturage. En nous référant aux apports de la psychologie et des normes sociales dans les modèles de prise de décision [9] et d'interactions stratégiques [10], nous formulerons plusieurs hypothèses répondant aux problèmes identifiés de masse critique, de motivation et de confiance.

Dans la section 1 nous proposerons une approche historique qui montrera l'évolution de la pratique $\mathrm{du}$ covoiturage et nous permettra d'identifier les facteurs de blocage de son développement. Nous donnerons également quelques exemples de modèles de coordination pour en montrer les limites. Dans une deuxième section, nous préciserons notre cadre d'expérimentation en nous appuyant sur la littérature comportementale.

\section{Le covoiturage : état des lieux, typologies et modèles d'interprétations classiques}

Comme nous allons le préciser rapidement ${ }^{7}$, notre champ d'étude est le covoiturage « domicile-travail ». Nous allons d'abord adopter une perspective historique pour établir le fondement de cette pratique. Dans cette lecture, le covoiturage est lié à une faiblesse du parc automobile. Dès que le taux d'équipement de la population a progressé, la pratique a presque disparu. Nous proposerons ensuite plusieurs typologies qui vont permettre d'établir la distinction avec le covoiturage « longue distance», et de révéler les limites des approches habituelles.

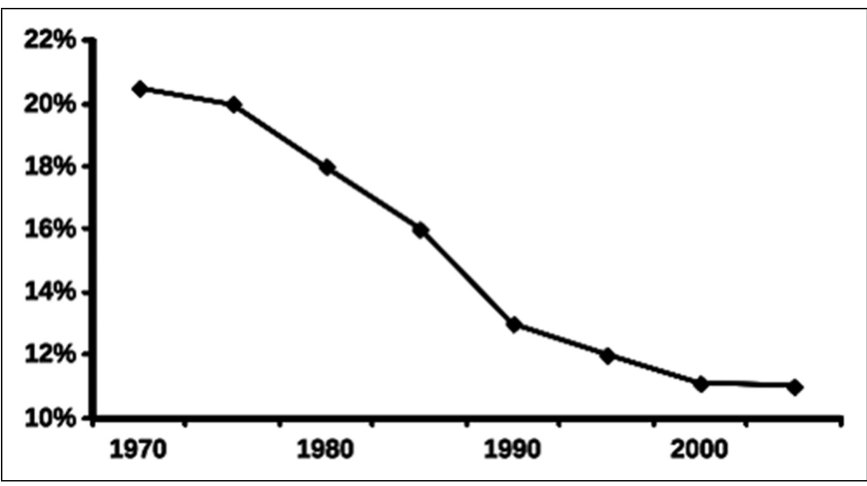

Fig. 1 Évolution du taux de covoiturage des travailleurs américains aux États-Unis entre 1970 et 2008 (d'après Chan et Shaheen, 2012)

\section{Développement historique et typologie}

\section{Le covoiturage :}

\section{une pratique ancienne en voie de disparition}

Avant tout, il est bon de rappeler que le covoiturage n'est pas une nouveauté qui pourrait décoller en même temps que d'autres pratiques de partage constitutives de ce qu'on appelle « l'économie collaborative » ou « l'économie positive $»^{8}$. Si l'on considère le covoiturage dans une perspective historique, il s'agit d'un phénomène qui n'a cessé de se réduire. On est ainsi passé d'une pratique qui touchait près de $30 \%$ des travailleurs américains en 1942 à environ $10 \%$ à l'heure actuelle [11], [12].

Selon Chan et Shaheen [12], on peut distinguer quatre grandes périodes historiques dans le covoiturage, animées par des paradigmes différents. L'effort de guerre (1940) fait appel au patriotisme et à la solidarité, la crise pétrolière (1970) aux économies d'énergie, la vague écologique (1990) aux débats sur la pollution, et enfin, la vague technologique depuis 2004 qui, en 2013, place beaucoup d'espoir dans le cloud et l'open data'.

La chute a été particulièrement brutale entre 1970 et 1990. "Le covoiturage est mort dans les années $1980 »$ remarquait Pisarski (1997). Effectivement, sur cette période, l'occupation moyenne des voitures individuelles est ${ }^{10}$ passée d'un taux de 1,18 à 1,09 aux États-Unis. Au Canada, Morency [11] observe la même désaffection en étudiant les enquêtes Origine-Destination de la région de Montréal réalisées en 1987, 1993, 1998 et 2003. Elle fait même remarquer que $70 \%$ des covoiturages sont en fait

\footnotetext{
${ }^{8}$ Voir le rapport du groupe de réflexion présidé par Jacques Attali : Pour une économie positive (La documentation française, 2013). ${ }^{9} \mathrm{Par}$ exemple, les projets européens : ECIM (European Cloud Intelligent Mobility) et OTN (Open Transport Net).

${ }^{10}$ Tiré de [6], les données 1990-2000 étaient extrapolées, mais se révèlent justes d'après les études plus récentes [12].
} 


\begin{tabular}{|c|c|c|}
\hline type de covoiturage & complexité de mise en œuvre & motivation principale \\
\hline longue distance & faible & économique \\
\hline domicile-travail & forte & \\
\hline spontané (HOV) & moyenne & pratique \\
\hline
\end{tabular}

Fig. 2 Typologie des formes de covoiturage (d'après Furuhata et al., 2013)

des déplacements familiaux, dont $15 \%$ ne concernent en fait que le passager : dépose d'enfants à l'école, etc. Les études les plus récentes (Chan et Shaheen [12]) ne montrent pas d'importants renversements de tendance, sinon des phénomènes saisonniers ou locaux, comme, par exemple, l'influence des $\mathrm{HOV}$ lanes ${ }^{11}$.

Cette perspective historique peut être résumée par le commentaire de Morency [11] : «Autrefois, on était forcé de partager sa voiture. Mais le développement des villes et la croissance économique ont offert à la population la liberté de se déplacer comme elle le veut. Être un passager est devenu une manière de se déplacer de moins en moins nécessaire et de moins en moins acceptable. ».

\section{Une typologie \\ pour caractériser les enjeux et les freins du covoiturage}

L'enjeu de la mobilité urbaine, tel que nous l'avons présenté en introduction, dépend de pratiques quotidiennes (trajets domicile-travail). Ce type de covoiturage doit être distingué du covoiturage longue distance et des offres de covoiturage à but commercial.

On distingue classiquement 3 types de covoiturage : « longue distance », « spontané », et « domicile-travail » (Furuhata [5]). Nous nous intéressons dans ce papier uniquement à la catégorie « domicile-travail », directement liée aux enjeux d'aménagement urbain, mais disons quelques mots sur les deux autres catégories.

Le covoiturage «longue distance » est le cas le plus répandu et le plus simple en termes de coordination puisque ses usagers sont plus flexibles que ceux des autres types de covoiturage en ce qui concerne à la fois les lieux de rencontre et les horaires. Ce type de covoiturage se rapproche des offres de transport à caractère commercial. En effet, il se distingue des autres catégories à la fois par son caractère exceptionnel et par le fait que le facteur économique constitue le facteur déterminant pour ses usagers (Furuhata [5]).

\footnotetext{
${ }^{11}$ High Occupancy Vehicule Lanes : voies de circulations réservées aux voitures avec passagers. Elles peuvent être permanentes ou liées à certaines tranches horaires, de type HOV-2 (un passager), HOV-3 (2 passagers) ou HOV-4. On parle également de HOT Lanes: High Occupancy Toll qui permettent l'accès aux « autosolistes » moyennant un péage.
}

En revanche, la catégorie «spontané » (qui intègre les HOV) permet d'éclairer notre sujet, et nous y reviendrons. Ce type de covoiturage souvent effectué avec des inconnus est riche d'enseignement sur l'établissement de la confiance nécessaire à cette pratique [7], [13].

\section{Approche traditionnelle et résultats empiriques}

\section{Le covoiturage : une affaire d'optimisation}

En ramenant le covoiturage à un problème complexe de coordination, les approches économiques multiplient les hypothèses implicites sur le comportement rationnel des participants (homo æeconomicus), posent la « masse critique de participants » comme unique déterminant, et prônent un avènement technologique permettant de mettre en œuvre les algorithmes proposés.

Le covoiturage tel qu'il est souvent présenté (voir les revues de littérature proposées par Agatz et al. [4], Shuldiner et Shuldiner [3], Huang et al. [14]) pose comme frein majeur à son adoption la difficulté d'organisation des trajets pour une masse critique d'usagers. Le développement du covoiturage est ainsi ramené à un problème complexe de compatibilité des trajets sur les variables temps et localisations. Cette vision favorise une approche principalement technologique du problème (Agatz et al. [14]).

Le trajet commun doit être défini à la fois de manière spatiale (il convient de trouver et d'optimiser une partie de trajet commune à plusieurs trajets), et temporelle (le trajet doit se faire dans des seuils de tolérance ${ }^{12}$ qui doivent pouvoir être acceptés par les différents usagers). Il y a également une contrainte d'échelle importante sur la coordination conducteur/passager. En effet, autant il apparaît possible d'imaginer une certaine souplesse pour un départ en vacances, autant la tolérance est faible pour les trajets quotidiens : celle-ci est inférieure à 5 minutes pour $75 \%$ des conducteurs interrogées dans une étude de Amey [15].

Cette idée peut se traduire par le schéma ci-dessous. Soit un conducteur (d) et un passager (r) avec les distances de déplacements séparées : $d_{v(d), w(d)}$ pour $(d)$ et $d_{(v(r), w(r)}$ pour (r), et des distances de coordination $d_{v(d), v(r)}$ et $d_{w(r), w(d)}$. On

\footnotetext{
${ }^{12}$ Temps passé à la coordination, trajet supplémentaire, détour, temps d'attente.
} 


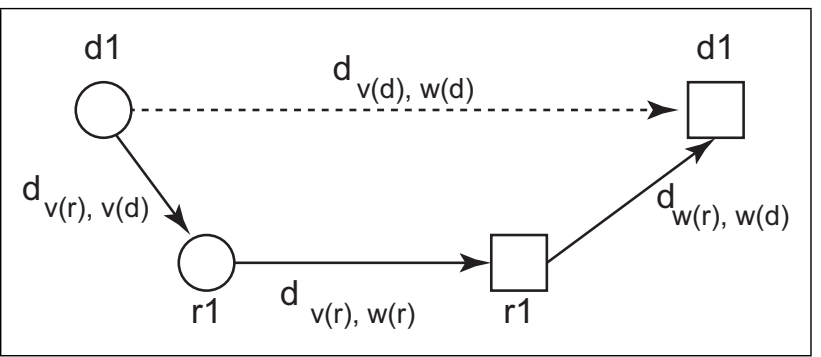

Fig. 3 Coordination simple un passager et un véhicule (d'après Agatz et al., 2012)

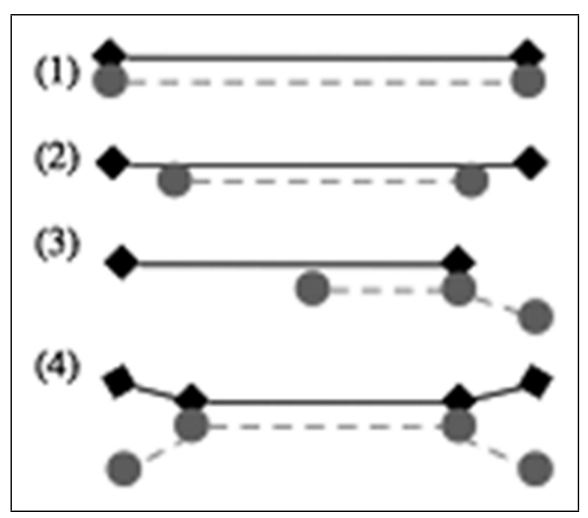

Fig. 4 Différents types de coordinations simples (d'après Furuhata et al., 2013)

voit qu'il n'y a un gain global que si $\mathrm{d}_{\mathrm{v}(\mathrm{d}), \mathrm{w}(\mathrm{d})}+\mathrm{d}_{(\mathrm{v}(\mathrm{r}), \mathrm{w}(\mathrm{r})}$

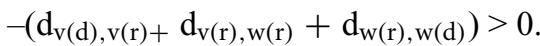

Ce qui est vrai pour des distances de déplacement très grandes par rapport aux distances de coordination des 2 participants, comme peut l'être un covoiturage longue distance de type Blablacar, semble l'être beaucoup moins en ce qui concerne des courtes distances.

En faisant varier les distances, ce modèle donne lieu à quatre types de covoiturages : 1. identique, 2. inclusif, 3. partiel, et 4. avec détour [5]. Cela donne donc huit cas d'études si l'on ajoute la condition de passagers uniques ou multiples.

Le problème est d'autant plus complexe quand on ajoute la dimension temporelle. Pour le type (1), il ne s'agit que de contraintes d'organisation personnelle, comme les heures de départ et de retour au travail. Mais pour les autres types : (2), (3) et (4), il faut également être capable de prévoir les temps des trajets de coordination afin de minimiser les attentes successives. La troisième dimension qu'il convient d'examiner est la maximisation théorique de participants au système. En effet (d) et (r) pourraient peut-être alternativement participer à d'autres coordinations, et ce n'est qu'avec une vue d'ensemble que l'on peut savoir lesquelles sont les plus bénéfiques.
Devant la complexité du modèle global, on pourrait être tenté de ne considérer que les cas simples, de type 1, mais cela reviendrait à restreindre le nombre de participants, ce qui est à l'opposé du but recherché. En effet, les modèles d'optimisation proposés nécessitent une masse critique d'usagers. En conséquence, la tendance va plutôt être de multiplier les situations, en considérant le cas de conducteurs prenant plusieurs passagers, ou de passagers combinant plusieurs covoiturages. Les propositions les plus récentes tournent autour du covoiturage dynamique, qui augmente le nombre de possibilités, mais nécessite un traitement algorithmique en temps réel [16].

C'est cette complexité assumée qui va faire espérer aux promoteurs du covoiturage une concrétisation de ces principes grâce à chaque nouveauté technologique : Internet, smartphones, GPS, big data, etc. Mais cet espoir tarde à montrer des résultats. Ainsi, cité par Furuhata et al. [5], un article du New York Times de 2011 remarquait que la promesse du développement du covoiturage grâce aux sites internet spécialisés était démentie par les chiffres, et que, de fait, la pratique de covoiturage continuait de reculer. En décembre 2013, c'est au tour de la très médiatique start-up Californienne RideJoy de jeter l'éponge, malgré un financement conséquent et un concentré de technologie mobile qui permet du covoiturage dynamique [17]. La raison invoquée est que, malgré une croissance satisfaisante, un nombre suffisant d'usagers ne semble pouvoir être atteint.

Nous faisions remarquer que ces traitements de coordination n'ont de sens qu'à partir d'une masse critique qui permet au système de tourner. La constitution de cette masse critique, pourtant fondamentale à tous les modèles discutés n'est que peu abordée. La motivation à faire du covoiturage est généralement considérée comme allant de soi et d'ordre économique. L'argumentation implicite est que l'augmentation des coûts de l'essence, et plus généralement des frais associés à la voiture individuelle, $\mathrm{y}$ compris les embouteillages et les péages urbains, devraient amener naturellement les conducteurs à vouloir partager ces frais en partageant leur véhicule.

Qu'il puisse y avoir des raisons autres qu'économiques au développement ou au ralentissement du covoiturage est pourtant constaté. Mais freins sociaux et psychologiques sont perçus comme des phénomènes exogènes au sujet, ou ramenés à leur plus simple expression. Il suffirait ainsi de préciser non-fumeur et de permettre aux femmes inquiètes de ne sélectionner que d'autres femmes comme covoitureurs pour résoudre ce point [14].

\section{Les limites de l'approche par l'optimisation}

Bien qu'elles ne soient pas prises en compte par les approches que nous venons de présenter, il existe beaucoup de données provenant de recensements ou d'enquêtes qui 


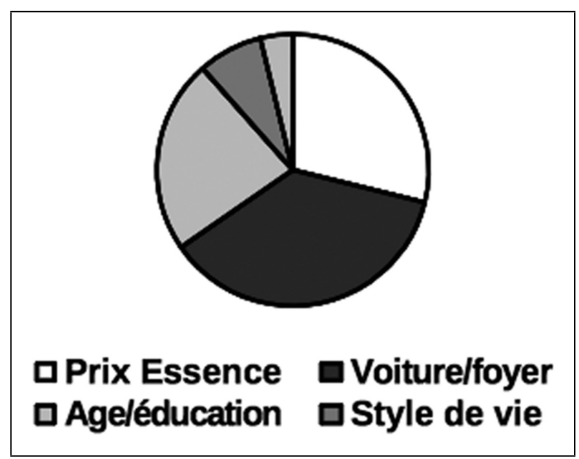

Fig. 5 Facteurs déterminants du covoiturage (d'après Ferguson 1997)

donnent une autre vision du sujet. Les données socioéconomiques montrent la limite des incitations financières, que nous pouvons par exemple comparer à celle de la hausse du tabac sur sa consommation. Ce qui pose la question de la motivation individuelle à covoiturer. Les études de psychologie sociale convergent sur plusieurs points, dont l'importance de la confiance entre les participants.

Pour une analyse économique et sociale du covoiturage, on se réfère généralement aux articles de Ferguson [6], de Morency [11], et Chan et Shaheen [12]. Ils concernent les États-Unis et le Canada, mais nous les compléterons par la suite avec des enquêtes concernant la France et l'Europe. Pour les aspects psychologiques, nous avons retenu quelques enquêtes significatives par l'étendue des questions abordées, notamment celle de Bonsall et al. [18], ou celles autour des pratiques de slugging ${ }^{13}$ d'Oliphant [13] et Burris et Winn [7].

L'impact limité des déterminants économiques. Bien que datant de 1997, l'étude de Ferguson [6] des facteurs impactant la courbe du covoiturage domicile-travail est très souvent citée, notamment du fait de la masse de données utilisées sur la période 1970-1990 : rapprochement des bases $d u$ Nationwide Personal transportation, de US Census of population, et du American Housing Survey. Notons tout d'abord que, déjà en déclin dans les années 1970, le covoiturage américain s'est effondré de $32 \%$ entre 1980 et 1990 . On peut souligner différents points. Tout d'abord que le coût de l'essence n'intervient que de façon mineure dans cet effondrement, puisque ni la crise du pétrole de 1979, ni l'augmentation du prix de l'essence dans les années 1980, (64 \% de hausse), n'ont empêché la chute du covoiturage de se poursuivre. D'après les calculs de Ferguson, la part économique ne représente que $30 \%$ des facteurs explicatifs de la chute du covoiturage. Avec une telle élasticité, il faudrait un levier énorme pour peser sur

\footnotetext{
${ }^{13}$ Covoiturage spontané autour des HOV lanes aux États-Unis.
}

les comportements. En effet, une augmentation régulière de près de $85 \%$ du coût de l'essence par décade serait nécessaire afin de seulement permettre de compenser le poids négatif des autres facteurs socio-économiques.

On pourrait ici faire une analogie avec les études sur la lutte contre le tabagisme. Ainsi, dans le rapport dédié par la Cour des Comptes à ce thème [19, p. 134], on note une élasticité de $0,3 \%$ du prix du tabac qui tombe même à $0,1 \%$ pour les augmentations inférieures à $10 \%$ en ce qui concerne la période de 2008 à 2012. Autrement dit, une augmentation du prix du tabac de $10 \%$ n'aurait un impact sur le volume consommé que de $1 \%$. On voit donc ici le dilemme de la politique de santé publique prise entre l'acceptation sociale de l'augmentation d'une nouvelle taxe et son efficacité.

Quant aux gains espérés pour le conducteur offrant un covoiturage, les avis divergent sur son évaluation et sa répartition [14]. Pour résumer, selon Agatz et al. [14], on peut trouver trois propositions différentes de répartition des dépenses. La première consiste en une répartition égale entre covoitureur et covoituré, la seconde en une répartition au prorata des distances, la troisième en une forme de prix de marché s'établissant par enchères entre les parties. Notons aussi le chevauchement vers le haut avec le marché professionnel de ce qu'on appelle le VTC (véhicule de tourisme avec chauffeur), dont la société Uber est actuellement l'exemple le plus représentatif. En 2013, la revente de Zimride, un des acteurs majeur du covoiturage en Californie, et le lancement par ses fondateurs de Lift, qui correspond à un concurrent low cost de Uber a montré la porosité entre les deux segments et la vulnérabilité du premier.

L'explication sociologique du déclin du covoiturage. $\mathrm{La}$ deuxième constatation de l'étude de Ferguson [6] est que, malgré deux décennies d'étalement urbain, l'éloignement géographique des habitations dans les banlieues qui disperse les trajectoires n'est pas une raison majeure de la baisse constatée du covoiturage. En effet, cet éloignement géographique n'explique que $4 \%$ de la baisse constatée.

Les trois principaux facteurs socio-économiques relevés sont : la possession d'une voiture, ou plus exactement le ratio nombre de voitures et la taille de la famille (38\%), le nombre d'actifs par foyer, et la combinaison âge et éducation $(24 \%)$. Le style de vie et l'évolution sociale comptent pour $8 \%$.

Il faut noter aussi le fait que le covoiturage urbain concerne avant tout les membres d'une même famille (Intra Household Ridesharing - IHHR ${ }^{14}$ ) : $70 \%$ en volume d'après une étude canadienne [11], ce qui rejoint les chiffres présentés par l'Ademe [2] en France.

\footnotetext{
${ }^{14}$ Intra Household Ridesharing : la personne transportée est du même foyer que le conducteur (conjoint, enfants...).
} 


\section{Identifiés mais peu étudiés : les freins psychologiques}

Bien que peu étudié, l'aspect psychologique du comportement semble être un déterminant important du potentiel de covoiturage des populations concernées.

Par exemple, une étude sur le potentiel de covoiturage du campus du MIT [15] montre un gain théorique en miles compris entre $9 \%$ et $27 \%$ du total des participants suivant la tolérance du conducteur à faire des détours pour aller chercher son passager. Dans la même étude, on note que $50 \%$ à $70 \%$ des personnes concernées pourraient covoiturer suivant les compatibilités géographiques et temporelles constatées alors qu'elles ne sont que $8 \%$ à le faire dans la réalité. Pour expliquer le faible développement du covoiturage malgré ces bénéfices potentiels, ces études mettent en évidence plusieurs facteurs psychologiques constituant un frein au covoiturage.

En premier lieu, les personnes interrogées craignent la perte de la flexibilité et de l'autonomie apportées par l'automobile. Ce point avait été relevé dès 1976 [20]. « Être maître de sa situation ${ }^{15}$ est aussi une des premières raisons invoquées par les conducteurs de l'enquête d'Oliphant [13].

Cela se traduit aussi pour le passager, par le refus d'accepter un covoiturage le matin sans garantie de voyage de retour le soir [2].

L'acceptation, ou le refus, du contact social constitue également une condition primaire. En effet, ce contact social peut être soit une motivation, le covoiturage permettant d' « avoir quelqu'un avec qui parler », soit, au contraire, un frein, le covoiturage ne permettant pas de satisfaire un « besoin d'être tranquille ». Pour l'étude réalisée par Bonsall et al. [18] d'un dispositif public sur quatre villes en Angleterre, il s'agit d'une condition initiale de participation.

La même étude montre qu'au bout de six mois, ce sont principalement les personnes qui se connaissaient déjà avant la mise en place de la plateforme de covoiturage qui continuent à le pratiquer. Et les plus réguliers sont ceux qui se covoituraient déjà auparavant de manière informelle.

$\mathrm{Ce}$ lien préexistant entre covoitureurs se retrouve également dans l'enquête réalisée par Chaube et al. [8] dans une petite ville universitaire américaine. Seulement $7 \%$ des personnes interrogées étaient prêtes à covoiturer avec un inconnu, même si la mise en relation se faisait par un site de covoiturage.

Ces dernières remarques sur la relation conducteurpassager nous amènent à évoquer l'importance de la confiance et la sécurité pour pratiquer le covoiturage. Pour préciser ce point, on peut s'intéresser aux études sur le covoiturage informel (slugging) autour des HOV lanes qui mettent en jeu un nombre important de personnes, dont une forte proportion (de l'ordre de $60 \%$ ) ne se connaît

${ }^{15}$ Like to be in control of my situation, cité par $34 \%$ des répondants. pas au préalable [13] et [7]. L'inquiétude est bien présente dans cette pratique, y compris chez les habitués, puisqu'elle arrive en deuxième raison citée du refus d'un covoiturage, la première raison étant la trop grande attente liée à cette pratique. Une solution conseillée, et de plus en plus fréquemment mise en œuvre, est de favoriser les HOV-3, c'est-à-dire d'imposer au moins 2 passagers pour bénéficier de l'accès aux files réservées, l'inquiétude étant, de plus, particulièrement ressentie dans le cas d'un covoiturage à un seul passager.

Cette inquiétude est liée à des risques réels et à des vides juridiques en cas de litige, d'accident ou d'agression. Aux États-Unis, les start-ups qui se sont positionnées sur le créneau du covoiturage sont régulièrement critiquées pour les risques qu'elles font prendre à leurs participants en organisant des covoiturages avec des inconnus. Il suffit par exemple de lire la chronique juridique du San Francisco Examiner qui s'intéresse régulièrement à ce sujet (Dolan [21]) : «Lift prend $20 \%$ des "dons" effectués par le passager au conducteur ». Ce n'est pas une cause sociale, c'est un business qui se drape dans un concept vert, futuriste et progressiste. Et en participant à ce business model, vous abandonnez les droits et protections que vous avez quand vous prenez un taxi...». Et, plus loin dans l'article, en parlant des conditions d'utilisations : « Ayant eu l'occasion de représenter des centaines de clients impliqués dans des accidents de la route, je peux dire qu'une telle tentative d'échapper à sa responsabilité est la plus détestable des pratiques commerciales. »

\section{Hypothèses expérimentales}

Les études focalisées sur l'optimisation des coordinations conducteur-passager échouent à expliquer la stagnation du covoiturage en deçà d'une masse critique d'usagers et semblent incapable de proposer des solutions aux freins constatés de la confiance et de la motivation des populations concernées. Le constat de la rationalité limitée des individus a amené l'économie à proposer de nouvelles approches intégrant des apports de la psychologie expérimentale et de la psychologie sociale [22], [23]. L'utilisation de ces nouveaux cadres d'étude nous permet de proposer plusieurs hypothèses de réponses au problème du faible développement $\mathrm{du}$ covoiturage. Ces hypothèses feront l'objet d'expérimentations de terrain (field experiment) ${ }^{16}$ dont nous souhaitons expliciter le fondement théorique.

\footnotetext{
${ }^{16}$ Ce type d'expérimentation en économie se distingue des expériences de laboratoire par la nature des participants, le réalisme des enjeux et le choix d'un contexte existant (d'après [24]). Il s'agit bien sûr ici d'un contexte de covoiturage domicile-travail avec une population recrutée dans une zone géographique déterminée.
} 


\section{Pour une approche comportementale du covoiturage}

\section{L'échec}

\section{de l'hypothèse rationnelle des modèles d'organisation}

Nous avons constaté que les approches classiques comportaient plusieurs failles.

En premier lieu, la faiblesse d'analyse des motivations des participants. La motivation d'ordre financière est régulièrement démentie par les retours de terrain et les simulations. En effet, les simulations très poussées sur le prix du pétrole [6] montrent une élasticité trop importante pour avoir un réel impact sur les pratiques. En ce qui concerne le partage des frais, il est peu adapté et rarement pratiqué sur des trajets quotidiens. La fixation d'un prix se heurte à plusieurs obstacles qui sont : le faible coût des alternatives quand elles existent (transports en communs souvent payés forfaitairement), le montant d'un trajet qui peut paraître dérisoire s'il n'inclut que le coût marginal (essence), ou trop important s'il se base sur un coût global d'amortissement du véhicule. Si on écarte l'aspect financier, l'argument qui revient le plus souvent est l'argument écologique (par exemple $69 \%$ des personnes interrogées [8]), mais malheureusement, ce point ne semble avoir que peu d'impact sur l'engagement des personnes concernées. La traduction en motivation et en action est sûrement un des enjeux majeurs de ce point.

La seconde faille des modèles proposés est qu'ils ne fonctionnent qu'à partir d'une masse critique d'utilisateurs qui jusqu'à présent leur fait défaut. Nous avons déjà abordé ce point, mais ajoutons que la faiblesse de l'offre est la première cause d'abandon du covoiturage par des personnes inscrites ( $80 \%$ des répondants de l'enquête Atema [2]).

Enfin, rien n'est déduit des aspects comportementaux, pourtant clairement identifiés. En premier lieu, que faire de la problématique de confiance qui apparaît indirectement dans la nature des covoiturages (famille, proches, relations établies) mais aussi plus directement : seulement $7 \%$ d'acceptation de covoiturage avec des inconnus [8], la crainte d'un mauvais covoiturage cité comme deuxième raison d'abandon de la pratique pour les HOT Lanes [7]. . .

Notons en passant que non seulement l'aspect comportemental n'est en général pas pris en compte dans les modèles, mais que même l'hypothèse rationnelle ne permet pas à ces modèles de fonctionner. Prenons un exemple pour illustrer ce point développé à partir d'une remarque de Agatz et al. [14].

Sur le schéma, nous voyons 2 conducteurs $\mathrm{d} 1$ et $\mathrm{d} 2$, et 2 passagers $\mathrm{r} 1$ et $\mathrm{r} 2$ avec les distances à parcourir identiques pour tous $(6 \mathrm{~km})$ et les distances de coordination que l'on imagine égale au départ et à l'arrivée. R1 est à $2 \mathrm{~km}$ de $\mathrm{d} 1$, de même que d2 et $\mathrm{r} 2$.

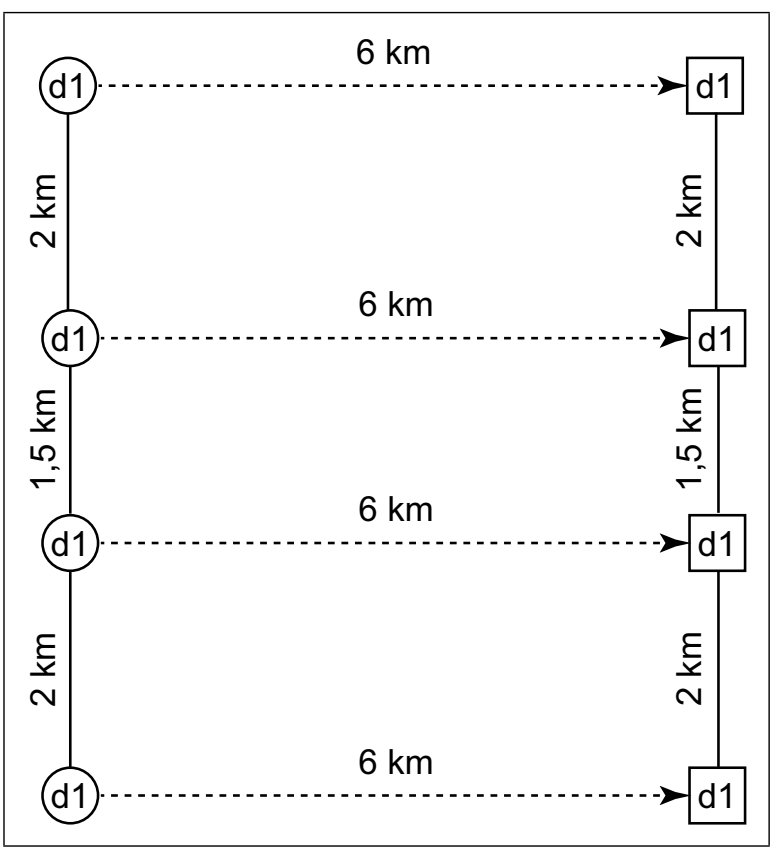

Fig. 6 Rationalité et coordination (d'après un exemple de Agatz et al, 2012)

La solution de moindre kilométrage (collectivement la plus économique) est de $20 \mathrm{~km}$ en associant $(\mathrm{d} 1, \mathrm{r} 1)$ et $(\mathrm{d} 2, \mathrm{r} 2)$.

Mais remarquons maintenant que $\mathrm{d} 1$ et $\mathrm{r} 2$ ne sont distants que de $1,5 \mathrm{~km}$, il est plus intéressant pour ces deux participants de covoiturer ensemble $(9 \mathrm{~km})$, même si cela laisse $\mathrm{r} 1$ et $\mathrm{d} 2$ dans une situation peu optimale puisqu'il leur faut parcourir $5,5 \mathrm{~km}$ pour se retrouver. La solution collective $(\mathrm{d} 1, \mathrm{r} 2)$ et $(\mathrm{d} 2, \mathrm{r} 1)$ est alors de $26 \mathrm{~km}^{17}$, pire que sans covoiturage $(24 \mathrm{~km})$.

On voit ici que l'hypothèse d'agent rationnel ne suffit donc pas à atteindre une solution collective optimum, et que le comportement mis à la porte du modèle d'étude revient par la fenêtre!

\section{Application au covoiturage : les trois hypothèses d'un programme de recherche expérimental}

Nous posons ici trois hypothèses qui pourraient faire l'objet d'un programme de recherche d'économie expérimentale s'appuyant sur des expériences de terrain. Nous allons justifier ces hypothèses en rapprochant des apports théoriques d'auteurs s'intéressant au comportement comme

\footnotetext{
${ }^{17} \mathrm{Si} \mathrm{r} 1$ dispose d'une voiture, ni lui ni d2 n'a intérêt à covoiturer (17 au lieu de 26), la solution globale sera donc $\mathrm{d} 1+\mathrm{r} 2+\mathrm{d} 2+\mathrm{r} 1$, soit 21 , ce qui reste inférieur à l'optimum.
} 
D. Kahneman, B. Frey, R. Boyer et A. Orléan avec des exemples pris dans notre bibliographie et pour certains déjà développés dans notre première partie.

La première hypothèse est qu'en raison de la composante essentielle de la confiance, le passage à grande échelle nécessaire aux gains collectifs ne peut pas être réalisé par des campagnes de recrutement de masse. Il faudrait plutôt s'orienter vers une addition progressive de «petits mondes », ce que nous allons montrer à la fois par des approches inspirées de la théorie des jeux [10] et de la Théorie de l'identité sociale (TIS) confortées par des exemples tirés de notre bibliographie.

La deuxième hypothèse est que la structure des motivations individuelles devrait être étendue et étudiée en contexte. L'hypothèse de rationalité amène à imaginer des gains privés sans commune mesure avec les efforts consentis, comme nous l'avons vu avec l'impact inexistant des chocs pétroliers. Nous proposons ici de comparer, dans le contexte du covoiturage, une gamme de motivations internes et externes, selon une typologie proposée par Ryan et Deci [25], reprise par Frey [26]. Nous nous inspirerons également des études sur les comportements pro-environnementaux (PEB - Pro Environment Behavior) [27].

Le troisième point concerne l'effet négatif sur l'engagement individuel de la présence manifeste d'une autorité. Ceci se caractérise principalement au niveau du discours, du cadre proposé et de la présence de contrôles. Cette troisième hypothèse devrait être traitée de manière préalable aux expérimentations proposées, puisqu'elle s'applique également au protocole d'expérimentation en réponse aux deux questions généralement posées aux expériences de terrain [28]: la neutralité du contexte expérimental sur le terrain (validité externe) et le contrôle des données collectées (validité interne).

\section{Première hypothèse : atteindre une masse critique d'usagers par addition de "petits mondes »}

Afin de poser clairement les alternatives et les gains attendus par les participants, commençons par représenter le problème d'atteinte de la masse critique sous la forme d'un jeu de coopération. Puisque cela nous place dans le contexte de la théorie des jeux, remarquons tout de suite que ce type de coordination qui relie des niveaux macro et micro [29] est extrêmement difficile à résoudre puisque, comme nous venons de le voir :

1. le comportement de chacun a des impacts sur tous les autres (externalité),

2. l'issue d'une action dépend des choix des autres : c'est le problème de «bison futé », si samedi est classé rouge

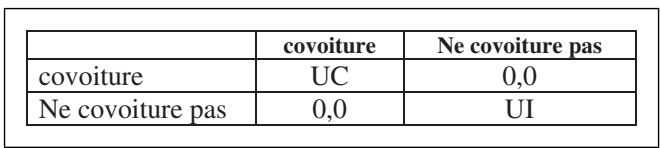

Fig. 7 Matrice du « jeu de covoiturage » (d'après l'auteur)

et que tout le monde décale son retour à dimanche, le résultat devient négatif, et enfin,

3. le gain principal est un gain collectif (macro) alors que l'on joue à un niveau individuel (micro).

Considérons donc pour la population de conducteurs un jeu à deux choix : « je covoiture » / « je ne covoiture pas », avec deux résultats stables : «nous covoiturons » ou «nous ne covoiturons » pas et deux états de frustrations correspondant à une coordination ratée.

$\mathrm{Si}$ on considère que l'Utilité d'un covoiturage (UC) est supérieure à l'Utilité d'un voyage individuel (UI), le covoiturage « raté » étant encore inférieur (0) : temps perdu à chercher un partenaire ou rendez-vous manqué, ou encore voyage désagréable...

(Notons au passage que cette représentation un peu frustre n'est cependant pas si éloignée que cela de la réalité). $\mathrm{Si}(\mathrm{p})$ est le pourcentage de conducteurs prêts à covoiturer, on voit qu'il existe un seuil $\mathrm{p}^{*}$ en deçà duquel $p^{*} \times$ UC $<(1-p) \times$ UI, autrement dit, en dessous d'un seuil minimum de participants, on a plus à gagner à ne pas essayer de covoiturer. À partir du moment où ce résultat devient une connaissance commune, le scénario « covoiture » va tout simplement disparaître, dominé par le scénario individuel. Mais si cette présentation, pour le moins défaitiste, était exacte, comment expliquer les pratiques, marginales mais constatées, de covoiturage. Autrement dit, en langage de la théorie des jeux : comment un scénario dominé peut-il survivre, et (ce qui nous intéresse encore davantage), pourrait-il se développer.

Boyer et Orléan [10] ont proposé une réponse originale à cette question. Il s'agissait d'expliquer la mise en œuvre des méthodes japonaises dans l'industrie automobile en Angleterre et aux États-Unis dans des contextes dominés par d'autres conventions (taylorisme, travail à la chaîne...). Leur approche a par la suite été discutée et enrichie [30], [31].

Les auteurs partent du principe que tous les participants ne sont pas en interaction avec tous les autres, il se forme naturellement des proximités, sociales ou géographiques, et à la frontière de ces espaces, des frontières ou zones de moindre interaction qui vont permettre la naissance de conventions alternatives au sein de sous-ensembles, et leur coexistence avec d'anciennes formes. Dans le schéma ci-dessous on voit une convention majoritaire (a) appliquée par des agents $(\mathrm{a}, \mathrm{a}, \mathrm{a} . .$.$) créant une utilité Ua, on représente$ 
la convention (b) appliquée par d'autres participants (b,b,b...) et créant une valeur supérieure Ub. On voit que la perte d'utilité du système produite par l'interaction des deux populations peut être inférieure au gain produit par la nouvelle convention si les participants $(b, b, b \ldots)$ sont regroupés et si les interactions entre populations ( $\mathrm{a}, \mathrm{a}, \mathrm{a} \ldots)$ et $(b, b, b . .$.$) sont limitées.$

La clé va donc être de chercher des « différenciations sociales ou culturelles qui permettent de localiser les effets de coordinations » $[10]$.

Le rapport entre groupes sociaux et conventions avait été relevé par Bessis et al. [32] à travers la lecture des travaux de H. Tajfel et de J. C. Turner sur l'identité sociale, l'auto-catégorisation et les groupes restreints [33, 34]. La catégorisation sociale, quelle qu'elle soit, va provoquer un rapprochement entre les personnes concernées et une différentiation renforcée avec les personnes non caractérisées par la catégorie active. Ainsi dans un salon professionnel deux « chercheurs » vont sympathiser en se détachant des « industriels », mais dans un laboratoire de recherche, deux chercheurs « économistes » vont se détacher des « sociologues».

En tout état de cause, notre hypothèse est donc qu'au lieu d'étendre une base d'utilisateurs en vue d'atteindre une masse critique, nous proposons plutôt de consolider des « petits mondes » déjà en place, puis de les multiplier. Cherchons à trouver dans la proximité : géographique, sociale et générationnelle le moyen de former de tels groupes. Nous rejoignons ici les travaux de Rallet et Torre [35] qui distinguent proximité géographique et proximité organisée ; cette dernière, de qualité relationnelle « offre à ses membres la capacité d'interagir ». D'après les auteurs, la proximité organisée s'explique ainsi, soit par une logique d'appartenance (à une entreprise, à une communauté), qui entraîne des habitudes d'interactions, soit par une logique de similitude, les individus partageant alors un même système de représentation, qui permet par exemple un langage commun.

Plusieurs études nous montrent la validité de cette approche, dont certaines que nous avons déjà citées comme celle d'Oliphant [11] qui notait que le covoiturage est une affaire principalement de famille ou de proches. Mais on peut également remarquer des pratiques de coopération se développer dans des groupes homogènes. Lovejoy et Handy [36] ont ainsi étudié les pratiques de partage de véhicules et de covoiturages dans des communautés d'immigrés mexicains aux États-Unis. Les raisons qui font que des personnes ne disposent pas de véhicules (en général des immigrés récents avec des ressources limitées ou sans papiers), s'étendant à leurs proches, c'est souvent un deuxième ou troisième niveau de lien social qui est sollicité, l'appartenance à la communauté étant alors un élément fort d'établissement de confiance. On peut aussi citer l'étude réalisée par Shergold et Parkhurst [37] sur

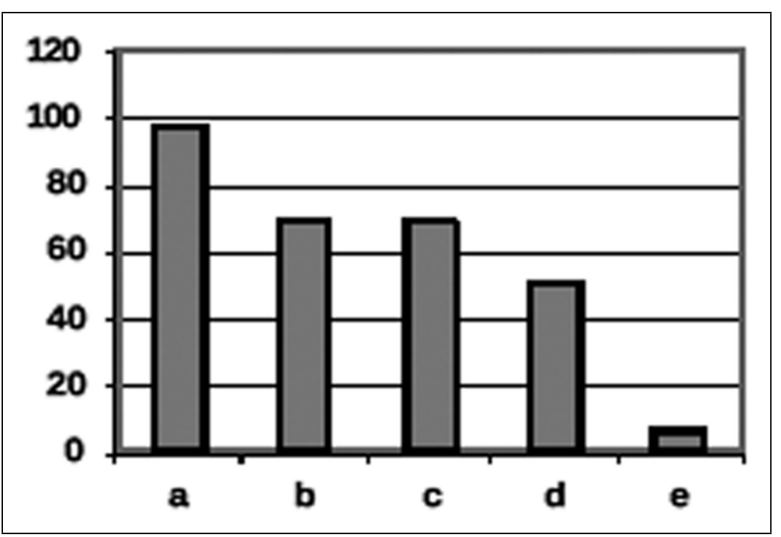

Fig. 9 Confiance en fonction du type de relation sociale (d'après Chaube et al., 2010)

les pratiques de transport dans des communautés rurales en Écosse, qui montre que le covoiturage est très présent dans les petits villages où les liens sociaux sont restés très importants. Charles et Kline [38] utilisent le covoiturage comme indicateur de mesure du " capital social », se basant sur les données de recensement, ils montrent que cet indicateur est plus important dans zones ethniquement homogènes.

Nous avons déjà cité l'enquête réalisée par Chaube et al. [8] dans une ville universitaire américaine pour étudier le rapport entre groupe et confiance. Parmi leur échantillon $65 \%$ des personnes interrogées font partie d'au moins une association ou communauté (sportive, étudiante, religieuse). À la question « avec qui accepteriez-vous de covoiturer s'il vous le proposait ? », $98 \%$ répondent positivement s'il s'agit d'un ami (a), $69 \%$ d'un ami d'ami (b), $69 \%$ d'un membre de leurs communautés (c), $50 \%$ d'un membre de la communauté universitaire (d), et seulement $7 \%$ d'un inconnu (e). Les résultats pour les conducteurs étaient tout à fait du même ordre $(99,80,80,60,10)$.

Notons que dans les exemples que nous venons de citer (immigrants, population rurale, ville universitaire), la notion de communauté est chaque fois suggérée par l'enquête. Si l'on veut s'appuyer sur les groupes sociaux pour étendre des pratiques, il faudra donc que ce soit au sein de dispositifs qui rendent manifestes ces catégorisations. Cependant, le lien établi par Turner [34] entre catégorisation sociale et rivalité intergroupe (favoritisme, discrimination) nous amène à préciser ce point.

De nombreuses études ont exploré les liens entre l'appartenance à des groupes et le renforcement de comportements sociaux, comme Dawes et Messick [39], qui $\mathrm{y}$ voient une réponse aux «dilemmes sociaux $»^{18}$. Nous

\footnotetext{
18 « Il est plus rentable pour chaque individu de démissionner que de coopérer, mais tous s'en trouvent mieux si ils coopèrent que s'ils démissionnent $»$.
} 


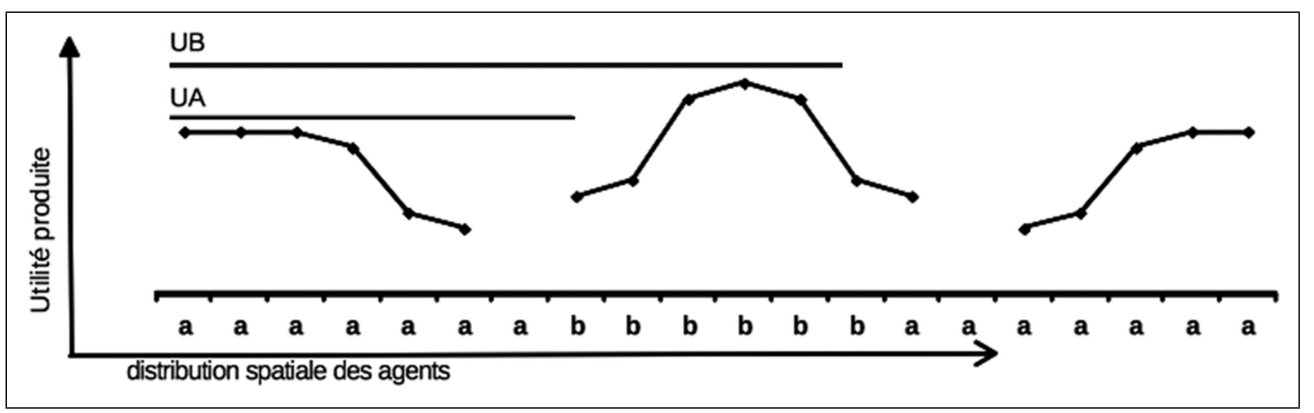

Fig. 8 Utilité différenciée d'un sous-groupe d'après (Boyer et Orléan, 1992)

citerons pour sa simplicité l'enquête de Wann et Polk [40] sur des étudiants de l'université de Murray. L'introduction dans un groupe testé des questions du type «Êtes-vous un fan de l'équipe de basket de l'école? » (test Sport Spectator Identification Scale-SSIS) ${ }^{19}$, augmentait considérablement l'échelle de confiance mesurée par des questions tirées du Doubt about the Trustworthiness of People Scale (DTPS) ${ }^{20}$ (par exemple : êtes-vous d'accord avec l'assertion suivante : «il est difficile de savoir à qui faire confiance de nos jours ».) Précisons qu'il s'agit d'une augmentation de la confiance envers les autres personnes en général, et non pas seulement envers les autres supporters de la même équipe. Il semble donc que l'on puisse, par identification sociale à un groupe, augmenter la confiance en général sans pour autant dégrader la relation avec les personnes d'autres groupes.

Pour résumer ce chapitre, nous avons constaté que présenté comme un jeu de coordination, le covoiturage, dominé en risque, ne permettait pas d'atteindre une masse critique. Cependant, nous avons présenté une solution dans laquelle des sous-ensembles de la population peuvent adopter de nouveaux comportements. Cette approche consistant à développer une pratique par la multiplication de « petits mondes » est confortée par la théorie de l'identité sociale ainsi que par la notion de «proximité organisée ». Nous avons également cité des résultats d'enquêtes reliant covoiturage et appartenance à des communautés. Ce point pourrait faire l'objet d'expérimentations comparant le taux de covoiturage de deux groupes, en provoquant pour l'un d'entre eux différentes manifestations renforcées de catégorisation sociale (sentiment d'appartenance à une communauté $^{21}$, distinction sociale...).

\footnotetext{
${ }^{19}$ Sept questions composent le Sport Spectator Identification Scale (SSIS; Wann \& Branscombe, 1993).

${ }^{20}$ Huit questions composent la partie Doubt about the Trustworthiness of People Scale (DTPS) de l'Échelle de mesure de satisfaction de la vie sociale (LFS) (Scheussler, 1982).

${ }^{21}$ On pourrait noter par exemple que les communications autour de la monnaie secondaire « Bristol pound » commencent en général par l'apostrophe « Nous autres, habitants de Bristol, nous sommes... » Voir le site : http://bristolpound.org.
}

\section{Deuxième hypothèse : étendre l'étude des motivations}

Quelles motivations amènent à covoiturer ? Nous avons vu que l'intérêt financier était souvent disqualifié par les études que nous avons citées ${ }^{22}$. La deuxième raison la plus souvent évoquée serait un intérêt pour l'écologie. Mais on sait que les déclarations ne valent pas actions, en particulier dans le domaine environnemental (Pelletier et Sharp [27]). Ainsi, les nord américains peuvent simultanément affirmer être conscients de l'impact de la consommation énergétique et augmenter celle-ci. En France, bien que la préoccupation écologique de la population soit une donnée vérifiée par les enquêtes d'opinion ${ }^{23}$, la performance environnementale des véhicules n'apparaît qu'en quatrième ou cinquième position derrière le prix, la performance, le confort et même l'esthétique dans les critères d'achat. ${ }^{24}$

Comment s'acquièrent de nouveaux comportements proenvironnementaux (Pro-Environmant Behaviour - PEB) ? Pelletier et Sharp [27] distinguent trois étapes : une phase de détection, une phase de décision et une phase d'implémentation. Nous ne rentrerons pas dans le détail de la première phase, qui concerne l'acceptation du message, en prenant comme présupposé que cet intérêt pour l'écologie est une donnée acquise, mais voyons à présent les deux phases suivantes.

\section{Phase de décision : la construction d'un cadre adapté à} l'action. La phase de décision nécessite pour l'individu de construire un cadre d'action (frame) reliant la problématique à son comportement. Par exemple face à deux personnes qui se battent, le comportement ne sera pas le même s'il s'agit

\footnotetext{
${ }^{22}$ Notons cependant les loteries mises en place par Prabakhar à Singapour et à Stanford pour inciter les usagers à éviter les heures de pointe [41] qui ont donné lieu a des résultats positifs et mesurables, mais selon nous, la nature des récompenses en font plus un jeu qu'une réelle incitation financière.

${ }^{23} 35-40 \%$ en ce qui concerne la pollution atmosphérique d'après les chiffres du ministère du Développement durable http://www.developpement-durable.gouv.fr/IMG/pdf/Rep_-

Chiffres_cle_environnement.pdf

${ }^{24}$ Ifop, Les Français et l'automobile, mai 2012.
} 
d'une compétition sportive, d'un jeu ou d'une agression (Goffman [42]). La construction de ce cadre se heurte souvent aux limitations cognitives de l'individu comme le remarque Thogersen [43]. Les travaux de Kahneman [44] permettent d'éclairer les processus mis en œuvre dans la création de ces cadres de décision. Ceux-ci ne se forment pas de manière logique et probabiliste, mais à partir d'informations de mémoire, de références subjectives et d'approximations. Il est par exemple facile de constater que la même personne aura une perception différente d'une augmentation de 300 euros selon qu'elle ne s'attendait pas à être augmentée ou qu'elle attendait une augmentation de 500 euros.

Dans le cas qui nous occupe, le comportement individuel se déroule à une échelle quotidienne, mesurée par des temps courts, des distances proches, alors que les enjeux (pollution, congestion) sont principalement présentés à une échelle collective. On voit la difficulté de relier les deux. Ainsi, en parlant de l'impact climatique de la consommation énergétique aux États-Unis, Thaler et Sunstein [45] déclarent : « il y a peu de chance qu'une personne qui met en marche l'air conditionné pendant quelques semaines pense à tout moment, ou même une fois par jour à la répartition des coûts personnels et coûts sociaux. ».

Une étude sur l'impact du message sur les dons caritatifs [46] montre que les donneurs sont plus réceptifs à une victime identifiée et à une action à leur portée (« avec 10 euros vous pouvez nourrir la petite Rokia pendant un mois ») que par une présentation argumentée et chiffrée ( nous avons besoin de 40 millions d'euros pour lutter contre la famine qui touche un million de personnes... »).

Nous voyons que rapporté aux enjeux de transport, cela nécessite de «traduire » l'impact collectif de pollution, ou les milliers d'heures perdues dans les embouteillages par une représentation à une échelle individuelle et quotidienne qui fasse sens, et qui intègre également l'impact des actions désirées. En effet, c'est le deuxième point relevé, le cadre doit permettre de voir l'impact de son comportement, sous peine de ne pas terminer la boucle d'apprentissage, voire de provoquer un effet d'impuissance [47].

Illustrons par un exemple positif cette fois-ci : Mark Martinez, directeur de la centrale Edison de Californie du Sud a eu l'idée en 2007 de distribuer à ses usagers un globe lumineux qui change de couleur en fonction de la consommation : de vert quand celle-ci est modérée au rouge vif quand elle devient excessive. Ce dispositif a permis, en quelques semaines de réduire la consommation de la population équipée de presque $40 \%$ aux heures de pointe [48]. Résultat d'autant plus intéressant qu'il avait auparavant mis en place des systèmes d'alerte pour les consommateurs, par SMS, par mail, par appel téléphonique automatique, sans aucun impact mesurable. On voit ici l'impact d'une représentation visuelle comparée à des informations chiffrées et textuelles.

L'intérêt d'une représentation de ce type est qu'elle est suffisamment significative pour permettre l'action et la communication, voire la coordination entre participants, tout en permettant de multiples interprétations, là où un ensemble de règles rigides et peu adaptatives ne permettraient pas de prendre en compte l'ensemble des situations individuelles [49, Ch. 7]. On voit l'intérêt de cette approche pour le sujet du covoiturage, avec presque autant de cas et de contraintes qu'il y a de personnes concernées.

2. Phase d'implémentation : le «coup de pouce» qui crée l'habitude. La phase d'implémentation vise à la mise en œuvre du nouveau comportement et surtout à sa permanence. C'est l'un des points soulevés par Pelletier et Sharp [27] : dès l'arrêt des campagnes d'incitations, on note l'abandon rapide de nombreux PEB, avec un retour à l'état antérieur. Plusieurs raisons sont données pour expliquer cette difficulté. En dehors des contraintes structurelles et sociales qui dépassent largement le sujet, il s'agit principalement de limitations et de surcharges cognitives.

Pour Thogersen [43] : face à l'ensemble des sollicitations et des contraintes du quotidien, il est très difficile d'introduire une nouvelle habitude de comportement. On parle également de faiblesse de volonté quand le bénéfice attendu est différé par rapport à l'action. L'économie comportementale s'est également penchée sur les mécanismes qui facilitent ou préviennent les changements d'habitude. Kahneman et al. [50] relient l'aversion aux pertes au désir de statu quo, qui amène à choisir les options par défaut et encourage les comportements de procrastination.

Ainslie [51] met en évidence une décroissante hyperbolique temporelle de la valeur des utilités qui explique la difficulté à mettre en place des habitudes liées à des objectifs lointains, même si ceux-ci sont plus importants que leurs alternatives proches (SS Sooner Smaller > LL Later Larger). Ainsi, sur le schéma, la décision prise la veille de se lever à 7 heures va se faire déborder malgré sa plus grande valeur par l'envie immédiate de dormir une heure de plus ${ }^{25}$.

On peut trouver une application à ces travaux dans le domaine de la santé et du sport avec la vogue de $l^{\prime}$ '« automesure » (quantified self) ${ }^{26}$. Whitson [52] explique ainsi que les dispositifs techniques proposés par Nike ou Foursquare peuvent permettre à leurs utilisateurs d'atteindre

\footnotetext{
${ }^{25}$ Une des pistes de réponse proposée par l'auteur est de découper l'objectif final en des étapes plus proches valorisées en tant que telles. ${ }^{26}$ L'automesure consiste à utiliser des dispositifs technologiques souvent portés (wearable) pour capter des données sur son activité biologique, physique, mentale et de se servir de ce feedback « objectif » pour améliorer ses pratiques principalement de santé et sportive.
} 


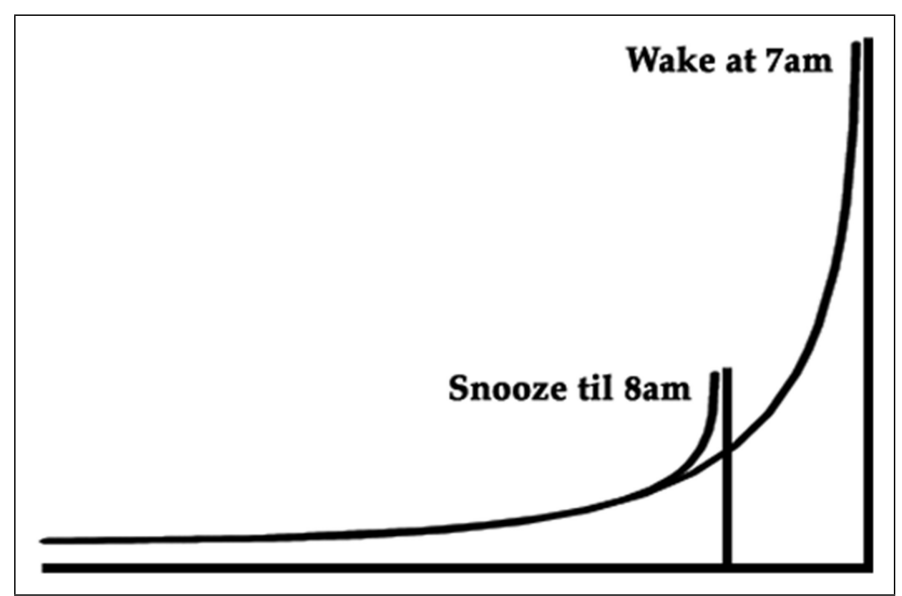

Fig. 10 Courbes hyperboliques temporelles des choix (d'après Ainslie, 2002)

des comportements souhaités en palliant les « faiblesses de la mémoire et aux fluctuations de l'intuition ». On va ainsi transformer l'objectif lointain et peu défini d' « être en forme » en des propositions d'actions concrètes et quotidiennes, des mesures et des gains donnant parfois lieu à des récompenses ou des badges. Pendant 6 mois, 5000 employés volontaires de la société Accenture équipés de podomètres ont ainsi participé à un jeu transformant les pas effectués en points et récompenses dans un objectif de santé (Gomes et al. [53]).

Thaler et Sunstein [45] ont proposé le concept de nudges (coup de pouce) pour qualifier toutes les techniques consistant à prendre en compte des biais cognitifs connus pour aider à des changements de comportement. ${ }^{27}$

\section{L'identité sociale comme facteur de changement de} comportement. Concluons ce thème des motivations en remarquant que l'identité sociale participe également à l'acquisition ou la modification des habitudes (Bessis et al. [32]). Ainsi, Wood et al. [55] ont profité du changement d'université d'étudiants pour évaluer des changements d'habitudes désirés : faire plus de sport, regarder moins la TV, lire les journaux. La conclusion de l'étude est que bien plus que l'intention initiale, ou la force de l'habitude antérieure, c'est le nouveau contexte social qui influe sur la capacité à changer (est-ce que le nouveau groupe regarde la TV, fait du sport ?). Une autre étude citée par Thalet et Sunstein [45] montre le lien entre la position choisie dans le dortoir par les étudiants et les résultats scolaires. En plus de son importance pour la confiance que nous avons évoquée

\footnotetext{
${ }^{27}$ Selon plusieurs études rassemblées sous le titre : Nudge, nudge, think, think, experimenting ways to change civic behaviour [54] sous la direction de Peter John, les nudges ne peuvent pas servir à faire prendre conscience ou à faire s'engager les individus dans un comportement civique, mais à les aider, une fois leur décision prise, à maintenir de nouvelles pratiques.
}

dans le chapitre précédent, le groupe social est également un puissant instrument de changement d'habitudes.

Ainsi, au-delà des classiques motivations monétaires ou de l'influence de l'information sur les comportements, nous avons soulevé trois nouvelles propositions : la représentation réflexive des enjeux collectifs, la mesure des actions, et l'identité sociale. Ces propositions semblent prometteuses d'après la littérature citée et nous avons présenté des applications variées dans les économies d'énergie, les dons caritatifs et le sport. Pour chacune de ces propositions, on peut facilement imaginer des dispositifs techniques (un compteur de CO2 économisé grâce au covoiturage du type du compteur du Téléthon par exemple) qui permettraient de vérifier leur efficacité dans le contexte du covoiturage quotidien.

\section{Troisième hypothèse : un contexte sécurisé sans autorité manifeste}

Parlons maintenant de la mise en place d'un environnement permettant la consolidation et la multiplication des « petits mondes » de coopération issus de l'approche que nous avons proposée. Une autorité manifeste et un discours de contrôle provoqueront un désengagement incompatible avec les ressorts de la coopération, on serait alors dans 1 '« amotivation » (crowding out) [25] et [26].

Mais s'il n'y a ni autorité, ni contrôle, il faut cependant protéger la confiance entre participants, la sécurité réelle et perçue, faute de quoi c'est un scénario dominant en risque qui va s'imposer. Coopération sans autorité, confiance sans contrôle : nous retrouvons ici le dilemme de l'arbitre interventionniste cher aux économistes. Débat d'ailleurs relancé par la proposition de Thaler et Sunstein [56] d'une politique encourageant les comportements civiques qu'ils appellent le « paternalisme libertaire ».

Durant la dernière décennie, l'établissement (et la disparition) rapide de communautés numériques massives : 
Facebook, Myspace, Linkedin, Foursquare... a permis de nombreuses recherches sur le sujet de la confiance. Dans une étude sur les tricheurs dans Foursquare, Glas [57] cite le cofondateur de la société, Dennis Crowley, qui oppose le plaisir de jouer aux contrôles nécessaires à celui-ci et explique que c'est tout l'enjeu des communautés virtuelles que de maintenir un équilibre entre ces deux pôles. Quand à Facebook, sa croissance s'est faite en deux étapes : pour la première, de 2004 à $2006^{28}$, il s'agissait d'un réseau uniquement réservé aux étudiants, ce qui lui a permis d'atteindre 10 millions d'utilisateurs actifs avec un taux de confiance très supérieur à son concurrent du moment Myspace [58]. Par la suite, Facebook a appliqué une politique de sécurisation ex post : la mise en place de nouveaux contrôles étant chaque fois justifiée par des infractions médiatisées. On retrouve ici le mythe très en vogue aux États-Unis qui voudrait que l'établissement de toute nouvelle communauté passe par une phase de «Far West » avant de se pacifier.

Comme c'était le cas pour le Facebook « première phase », notre proposition de croissance du covoiturage par adjonction de groupes simplifie les choses, au moins au départ. Alors que le problème de l'approche classique est d'établir la confiance entre personnes ne se connaissant pas, nous devons nous contenter d'étendre une relation de confiance déjà en place en la protégeant de dérives de comportements (abus) et d'introduction non contrôlée de nouveaux participants. En continuant de nous inspirer $\mathrm{du}$ développement des communautés numériques, nous proposons de nous appuyer sur deux mécanismes que sont le parrainage et la transparence sociale des actions.

\section{L'expansion de l'espace de confiance par le parrainage.} Depuis la mafia, jusqu'aux sociétés secrètes, le parrainage, ou cooptation a fait ses preuves pour étendre des communautés de manière sécurisée. On peut trouver un 29 exemple original dans la diffusion à partir de $1991 \mathrm{du}$ logiciel de cryptage : PGP (Pretty Good Privacy) proposé par P. Zimmerman ${ }^{30}$ pour protéger les individus des écoutes gouvernementales (ce qui lui a valu de sérieux problèmes). Une des particularités de PGP est que la certification des clés permettant le chiffrement, au lieu d'être réalisée par une autorité centralisée (ou au moins communément admise) comme c'est le cas dans d'autres systèmes, se fait de proche en proche : si Dave à confiance en Alice et qu'Alice se porte

\footnotetext{
${ }^{28}$ Jesdanun, A. (2006). Facebook to open to all Internet users. Associated Press, 11 septembre 2006 : $\mathrm{http}: / / w w w . b o s t o n . c o m / n e w s / e d u c a t i o n / h i g h e r / a r t i c l e s / 2006 / 09 / 11 /$ facebook_site_to_open_doors_to_anyone/?rss_id=Boston.com $+\% 2 \mathrm{~F}+\mathrm{News}$

${ }^{29}$ Illustration tirée de OpenPGP certificates http://gnutls.org/manual/html_node/OpenPGP-certificates.html

${ }^{30}$ Voir sur Wikipedia : http://fr.wikipedia.org/wiki/Pretty_Good_Privacy
}

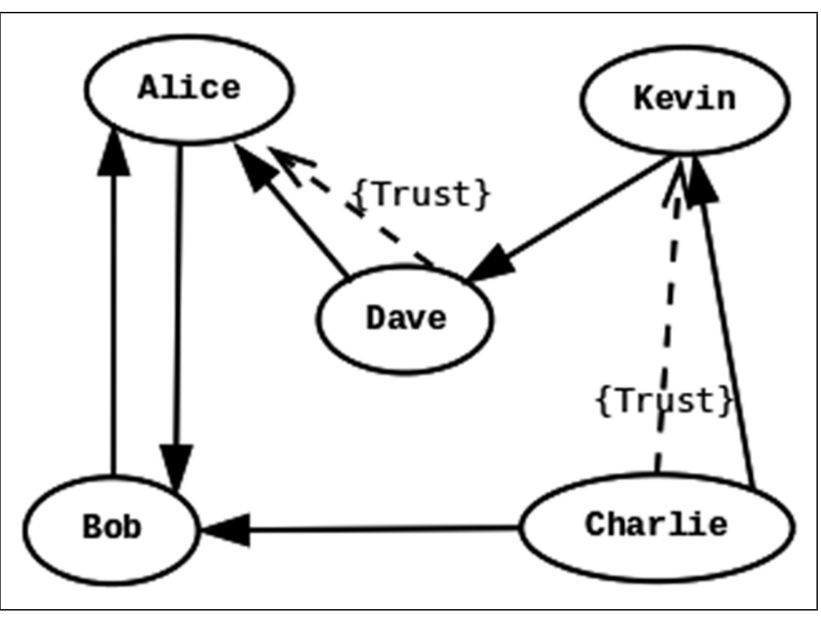

Fig. 11 Échange de clés dans PGP

garante de Bob, alors elle peut certifier la clé de Bob pour Dave, créant ainsi un nouveau lien sécurisé.

5. Le conformisme (contrôle social). En 1859, J. S. Mills ${ }^{31}$ évoquait déjà le conformisme comme étant la forme la plus absolue de contrôle de l'individu. En 1786, J. Bentham, avec son fameux panoptique montrait que le regard du surveillant n'était même plus nécessaire à partir du moment qu'il était manifesté. Le principe du contrôle par la transparence reste tout aussi efficace de nos jours. Voyons par exemple, comme l'a fait Pélegrin-Genel [59], l'impact de l'architecture sur les comportements des individus. Elle note par exemple que l'objectif des espaces ouverts, sans guichets, utilisés d'abord par les fast foods, mais de plus en plus par des services (comme La Poste), est de faire contrôler les employés par les clients eux-mêmes. Pélegrin-Genel parle « d'une gestion par le regard ». Difficile de faire une pause, ou de discuter avec un collègue sous l'œil de clients pressés dans des files d'attentes toujours trop longues. C'est également un argument pointé dans les open spaces : on remplace, souvent efficacement, la pointeuse par le regard des collègues « tu ne t'es pas réveillé ce matin?».

Avec une intentionnalité différente, et plus proche de notre sujet, ce principe démontre son utilité sur les réseaux sociaux. Guillory et Hancock [60] relèvent ainsi sur Linkedin un taux de triche sur les diplômes très inférieur au taux constaté sur les $\mathrm{CV}$ «papier » $(2,8 \%$ dans le test contre un taux constaté de 20 à $50 \%$ dans la plupart des études). Ceci est expliqué par le fait que chaque information du parcours professionnel est, dans Linkedin, visible par l'ensemble du réseau relationnel, et également corrélée avec toutes les personnes indiquant un cursus identique à la même période. Ce type de contrôle par référence nous semble

\footnotetext{
$\overline{{ }^{31} \text { De la liberté, }} 1859$.
} 
bien adapté au covoiturage qui implique par nature plusieurs personnes dans un groupe de personnes se connaissant (d'après notre hypothèse).

Comment sécuriser et contrôler la pratique du covoiturage sans mettre en place des dispositifs autoritaires qui provoqueraient des désengagements (crowding out) des personnes concernées ? Nous avons proposé trois pistes. La première consiste à une introduction progressive de mécanismes de contrôle, stratégie adoptée par les communautés en ligne. La deuxième propose une croissance des participants par parrainage permettant de s'assurer de la qualité des nouveaux entrants. La troisième se base sur le contrôle social avec des dispositifs visant à rendre publiques les déclarations et actions effectuées. Comme nous l'avons dit en introduction de cette partie, ces pistes peuvent également s'appliquer à la mise en place de protocoles expérimentaux en field experiment (recrutement, contrôle des résultats).

\section{Conclusion}

Après avoir rappelé que le covoiturage domicile-travail était une pratique devenue marginale, nous avons constaté que la plupart des études relatives au sujet ne proposaient qu'une approche algorithmique de résolution de la problématique de coordination, en ne prenant en compte les freins sociaux et psychologiques que sous formes de remarques. Nous avons proposé de tirer parti de nouvelles approches comportementales qui se proposent d'introduire des données sociologiques et psychologiques dans le champ de l'analyse économique.

Ceci nous a permis de formuler plusieurs hypothèses. La première est que l'atteinte d'une masse critique de participants pourrait se faire, non par cumul linéaire d'utilisateurs anonymes, mais par adjonction de groupes sociaux. Ce point est d'ailleurs corroboré par les descriptions du covoiturage tel qu'il est pratiqué aujourd'hui, à savoir dans un contexte essentiellement familial, de personnes proches, ou avec un lien communautaire marqué. La deuxième hypothèse concerne les motivations : si le terrain semble disqualifier l'incitation financière, l'engagement à des pratiques écologiques reste à traduire en actes. Nous avons proposé trois pistes pour y remédier : (i) trouver une représentation qui relie l'enjeu collectif à l'impact individuel (ii) permettre de mesurer le résultat des actions et (iii) s'appuyer sur des effets de mimétisme sociaux.

Notre troisième point qui concerne la sécurité peut s'appliquer aussi bien à la mise en œuvre de services de covoiturage qu'à un protocole expérimental sur ces mêmes pratiques. Nous avons évoqué plusieurs mécanismes, comme le conformisme ou le parrainage, permettant l'établissement de la confiance nécessaire aux comportements coopératifs, en évitant de recourir à une autorité de contrôle manifeste.

Ces différentes hypothèses sont à la base d'un programme de recherche pluriannuel (2015-2017) sur la zone du campus de Paris-Saclay.

Cette zone qui devrait à terme accueillir près de $15 \%$ de la recherche française souffre d'un double problème de transport : un engorgement des accès et des transports publics aux heures de pointes (matins et soirs) et un manque de transport intra-site en journée. Ces conditions devraient encore se dégrader dans les années qui viennent puisque de nouveaux établissements sont attendus alors que les infrastructures de transports les plus importantes ne seront pas encore opérationnelles [61].

Nous allons y réaliser plusieurs expérimentations de terrain autour des hypothèses que nous avons présentées dans ce papier, appliquées aux déplacements quotidiens des personnes habitant ou travaillant sur ce site. Nous espérons que ces travaux pourront participer au développement des pratiques d'entraide et de responsabilité citoyenne, comme le covoiturage, nécessaires à une croissance urbaine pour laquelle les transports représentent un défi majeur.

\section{Bibliographie}

1. Seto, KC, Güneralp, B, Hutyra, LR (2012) Global forecasts of urban expansion to 2030 and direct impacts on biodiversity and carbon pools, Proc. Natl. Acad. Sci. 109(40) : 16083-16088.

2. conseil, Atema (2010) Caractérisation de services et usages de covoiturage en France : quels impacts sur l'environnement, quelles perspectives d'amélioration ?, Agence de maîtrise de l'énergie.

3. Shuldiner, AT, Shuldiner, PW (2013) The measure of all things: reflections on changing conceptions of the individual in travel demand modeling, Transportation 40(6): 1117-1131.

4. Agatz, N, Erera, A, Savelsbergh, M, Wang, X (2012) Optimization for dynamic ride-sharing: A review, Eur. J. Oper. Res. 223(2): 295-303.

5. Furuhata, M, Dessouky, M, Ordóñez, F, Brunet, M-E, Wang, X, Koenig, S (2013) Ridesharing: The state-of-the-art and future directions, Transp. Res. Part B Methodol. 57: 28-46.

6. Ferguson, E (1997) The rise and fall of the American carpool: 1970-1990, Transportation 24(4): 349-376.

7. Burris, MW, Winn, JR (2006) Slugging in houston-casual carpool passenger characteristics, J. Public Transp. 9(5): 23, 2006.

8. Chaube, V, Kavanaugh, AL, Perez-Quinones, MA (2010) Leveraging social networks to embed trust in rideshare programs, in 43rd Hawaii International Conference on System Sciences (HICSS), pp. 1-8.

9. Kahneman, D, Tversky, A (1979) Prospect Theory: An analysis of Decision under Risk.

10. Boyer, R, Orléan, A (1992) How do conventions evolve?, J. Evol. Econ. 2(3): 165-177.

11. Morency, C (2006) The ambivalence of ridesharing, Transportation 34(2) 239-253.

12. Chan, ND, Shaheen, SA (2012) Ridesharing in North America: Past, Present, and Future, Transp. Rev. 32(1): 93-112.

13. Oliphant, M (2008) The Native Slugs of Northern Virginia, Master Sci. Urban Reg. Plan. Va. Tech Blacksbg. 
14. Huang, HJ, Yang, H, Bell, MG (2000) The models and economics of carpools Ann. Reg. Sci. 34(1): 55-68.

15. Amey, A (2011) Proposed methodology for estimating ridesharing viability within an organisation: application to the MIT community, Transp. Res. Board Annu. Meet. 11: 2585.

16. Pentico, DW (2007) Assignment problems: A golden anniversary survey, Eur. J. Oper. Res. 176(2): 774-793.

17. ehrenbacher, $K$ (2013) Another failed attempt to make ride sharing work in the U.S., Ridejoy to shut down, Gigaom.

18. Bonsall, PW, Spencer, AH, Tang, W-S (1984) What makes a carsharer?, Transportation 12(2): 117-145.

19. Cour des Comptes (2012) Les politiques de lutte contre le tabagisme, Rapport d'évaluation, Communication au président de l'Assemblée national pour le Comité d'évaluation et de contrôle des politiques publiques.

20. Dueker, KJ, Levin, IP (1976) Carpooling: Attitudes and participation, Technical report 81 .

21. Dolan (2013) Know the risks of rideshare services, The San Francisco Examiner. [Online] http://www.sfexaminer.com/sanfrancisco/know-the-risks-ofrideshare-services/Content?oid $=2482848$. [accessed 24 march 2014].

22. Orléan, A (2002) Le tournant cognitif en économie, Rev. d'Économie Polit. 112(5) : 717.

23. Petit, E (2011) L'apport de la psychologie sociale à l'analyse économique, Rev. d'Économie Polit. 121(6) : 797.

24. Harrison, GW, List, JA (2004) Field experiments, J. Econ. Lit.: 1009-1055.

25. Ryan, RM, Deci, EL (2000) Intrinsic and Extrinsic Motivations: Classic Definitions and New Directions, Contemp. Educ. Psychol. 25(1): 54-67.

26. Frey, BS, Jegen, R (2001) Motivation crowding theory, J. Econ. Surv. 15(5): 589-611.

27. Pelletier, LG, Sharp, E (2008) Persuasive communication and proenvironmental behaviours: How message tailoring and message framing can improve the integration of behaviours through self-determined motivation., Can. Psychol. Can. 49(3): 210-217.

28. Harrison, GW, List, JA (2003) What constitude a field experiment in economics, Field Experiments in Economics 10.

29. Cooper, R, John, A (1988) Coordinating Coordination Failures in Keynesian Models, Q. J. Econ. 103(3): 441.

30. Sugden, R 51995) The coexistence of conventions, J. Econ. Behav. Organ. 28(2): 241-256.

31. Shi, F (2013) Comment on "On the co-existence of conventions" [J. Econ. Theory 107 (2002): 145-155], J. Econ. Theory 148(1): 418-421.

32. Bessis, F, Chaserant, C, Favereau, O, Thévenon, O (2007) 11. L'identité sociale de l'homo conventionalis, Recherches : 181195.

33. Tajfel, H (1974) Social identity and intergroup behaviour, Soc. Sci. Inf., 13(2): 65-93.

34. Turner, JC (1975) Social comparison and social identity: Some prospects for intergroup behaviour, Eur. J. Soc. Psychol. 5(1) : $1-34$.

35. Rallet, A, Torre, A (2004) Proximité et localisation, Économie Rurale 280(1) : 25-41.

36. Lovejoy, K, Handy, S (2011) Social networks as a source of private-vehicle transportation: The practice of getting rides and borrowing vehicles among Mexican immigrants in California, Transp. Res. Part Policy Pract. 45(4): 248-257.

37. Shergold, I, Parkhurst, G (2012) Transport-related social exclusion amongst older people in rural Southwest England and Wales, J. Rural Stud. 28(4) : 412-421.

38. Charles, KK, Kline, P (2006) Relational Costs and the Production of Social Capital: Evidence from Carpooling*, Econ. J. 116(511): 581-604.
39. Dawes, RM, Essick, DM (2000) Social dilemmas, Int. J. Psychol. 35(2): 111-116.

40. Wann, DL, Polk, J (2007) The Positive Relationship between Sport Team Identification and Belief in the Trustworthiness of Others., North Am. J. Psychol. 9(2).

41. Merugu, D, Prabhakar, BS, Rama, NS (2009) An incentive mechanism for decongesting the roads: A pilot program in Bangalore, Proc. of ACM NetEcon Workshop.

42. Goffman, E, Joseph, I, Dartevelle, M, Joseph, P (1991) Les Cadres de l'expérience. Paris : Les Éditions de Minuit.

43. Thøgersen, J (2005) How May Consumer Policy Empower Consumers for Sustainable Lifestyles?, J. Consum. Policy 28(2): 143-177.

44. Kahneman, D (1992) Reference points, anchors, norms, and mixed feelings, Organ. Behav. Hum. Decis. Process. 51(2): 296-312.

45. Thaler, RH, Sunstein, CR (2008) Improving decisions about health, wealth and happiness. New Haven: Yale University Press.

46. Small, DA, Loewenstein, G, Slovic, P (2007) Sympathy and callousness: The impact of deliberative thought on donations to identifiable and statistical victims, Organ. Behav. Hum. Decis. Process. 102(2) : 143-153.

47. Abramson, LY, Seligman, ME, Teasdale, JD (1978), Learned helplessness in humans: critique and reformulation., J. Abnorm. Psychol. 87(1): 49.

48. Thomson, C (2007), Desktop Orb Could Reform Energy Hogs, Wired. [Online]. Available: http://www.wired.com/techbiz/people/magazine/1508/st_thompson. [Accessed: 23 March 2014].

49. Batifoulier, P, de Larquier, G Eds. (2001) Théorie des conventions. Paris : Economica.

50. Kahneman, D, Knetsch, JL, Thaler, RH (1991) Anomalies: The endowment effect, loss aversion, and status quo bias, J. Econ. Perspect.: 193-206.

51. Ainslie, G (2002) The effect of hyperbolic discounting on personal choices, in Annual Convention of The American Psychological Association.

52. Whitson, JR (2013) Gaming the Quantified Self, Surveill. Soc. 11.

53. Gomes, N, Merugu, D, O'Brien, G, Mandayam, C, Yue, JS, Atikoglu, B, Albert, A, Fukumoto, N, Liu, H, Prabhakar, B (2012) Steptacular: an incentive mechanism for promoting wellness, in Fourth International Conference on Communication Systems and Networks (COMSNETS), 2012, pp. 1-6.

54. John, P (2013) Nudge, nudge, think, think: experimenting with ways to change civic behaviour. London: Bloomsbury Academic.

55. Wood, W, Tam, L, Witt, MG (2005) Changing Circumstances, Disrupting Habits., J. Pers. Soc. Psychol. 88(6): 918-933.

56. Thaler, RH, Sunstein, CR (2003) Libertarian Paternalism, Am. Econ. Rev. 93(2): 175-179.

57. Glas, R (2013) Breaking reality: Exploring pervasive cheating in Foursquare, Trans. Digit. Games Res. Assoc. 1(1).

58. Fogel, J, Nehmad, E (2009) Internet social network communities: Risk taking, trust, and privacy concerns, Comput. Hum. Behav. 25(1): $153-160$

59. élegrin-Genel, E (2010) Des souris dans un labyrinthe. Paris : Les Empêcheurs de penser en rond- La Découverte.

60. Guillory, J, Hancock, JT (2012) The Effect of Linkedin on Deception in Resumes, Cyberpsychology Behav. Soc. Netw. 15(3): 135-140.

61. Établissement public de Paris-Saclay (EPPS) (2014) Présentation publique du 23 juin 2014, Enquête publique préalable à l'autorisation de réaliser les travaux de la Zone d'Aménagement Concerté du projet urbain de Moulon, Établissement public d'aménagement de Paris-Saclay. 
